\title{
Correction to: Intra-Arterial Transplantation of Low-Dose Stem Cells Provides Functional Recovery Without Adverse Effects After Stroke
}

\author{
Yuhtaka Fukuda ${ }^{1}$. Nobutaka Horie ${ }^{2} \cdot$ Katsuya Satoh $^{3} \cdot$ Susumu Yamaguchi ${ }^{2} \cdot$ Youichi Morofuji $^{2} \cdot$ Takeshi Hiu $^{2}$. \\ Tsuyoshi Izumo ${ }^{2} \cdot$ Kentaro Hayashi $^{2} \cdot$ Noriyuki Nishida $^{3} \cdot$ Izumi Nagata $^{2}$
}

Published online: 30 January 2020

๑) Springer Science+Business Media, LLC, part of Springer Nature 2020

\section{Correction to: \\ Cellular and Molecular Neurobiology (2015) 35:399-406 https://doi.org/10.1007/s10571-014-0135-9}

The original version of this article unfortunately contained an error in affiliation of Yuhtaka Fukuda.

The correct affiliation is given below:

Department of Neurosurgery, Graduate School of Biomedical Sciences, Nagasaki University, 1-7-1, Sakamoto, Nagasaki 852-8501, Japan

Publisher's Note Springer Nature remains neutral with regard to jurisdictional claims in published maps and institutional affiliations.

The original article can be found online at https://doi.org/10.1007/ s10571-014-0135-9.

Nobutaka Horie

nobstanford@gmail.com

1 Department of Neurosurgery, Graduate School

of Biomedical Sciences, Nagasaki University, 1-7-1,

Sakamoto, Nagasaki 852-8501, Japan

2 Department of Neurosurgery, School of Medicine, Nagasaki University, 1-7-1, Sakamoto, Nagasaki 852-8501, Japan

3 Department of Molecular Microbiology and Immunology, School of Medicine, Nagasaki University, Nagasaki, Japan 